\title{
Perceptual Attentional Set-Shifting Is Impaired in Rats with Neurotoxic Lesions of Posterior Parietal Cortex
}

\author{
Matthew T. Fox, Morgan D. Barense, and Mark G. Baxter \\ Department of Psychology, Harvard University, Cambridge, Massachusetts 02138
}

\begin{abstract}
The posterior parietal cortex (PPC) is believed to be involved in the representation of spatial information, including spatial attentional processing. Because the PPC is extensively interconnected with frontal cortical regions involved in attention and executive function, we sought to determine whether PPC was involved in nonspatial attentional processes such as those of the frontal areas to which it projects. Lesions of the medial frontal cortex (in rats) or lateral prefrontal cortex (in nonhuman primates) impair the ability to shift attention from one perceptual dimension of a stimulus to another (referred to as an extradimensional shift). Rats with neurotoxic lesions of the PPC tested in an attentional set-shifting paradigm demonstrated a pattern of impairment identical to that of rats with medial frontal cortex lesions: they were selectively impaired on the extradimensional shift phase of the task. Performance in other phases of the task was indistinguishable from that of control rats, including the ability to reverse a previously learned discrimination. These findings are consistent with models that assign the PPC a prominent role in cortical attentional processing networks, as well as a role for the PPC in processing information about expectancy and surprise. They also suggest, importantly, that the interaction between the PPC and the frontal cortex is not limited to spatial attentional processing.
\end{abstract}

Key words: attention; frontal cortex; posterior parietal cortex; rats; reversal learning; set-shifting

\section{Introduction}

Converging evidence from humans with brain damage (Halligan and Marshall, 1984; Posner et al., 1984; Petersen et al., 1989), functional neuroimaging studies of normal humans (Corbetta et al., 1993, 1995; Rogers et al., 2000), and lesion studies in nonhuman animals (Mesulam, 1981; King and Corwin, 1993; Bucci et al., 1998) indicates that the parietal lobes are necessary for attentional function. One longstanding view is that the parietal contribution to attention is limited to spatial functions. This is reinforced by the large number of studies using visual search testing paradigms as measures of attention (Robinson et al., 1995; Steinmetz and Constantinidis, 1995). However, few experiments have examined the contribution of the parietal cortex to nonspatial attentional function, or to executive function more generally.

The cortical network for spatial attentional function includes the frontal and parietal cortex (Mesulam, 1981). Anatomical evidence supports a role for these areas in attention. There are reciprocal projections between the posterior parietal cortex (PPC) and the frontal cortex in both rodents and primates (Selemon and Goldman-Rakic, 1988; Andersen et al., 1990; Reep et al., 1994). Unilateral lesions of either the PPC or the medial agranular (AGm) cortex produce multimodal hemifield neglect in rats (King and Corwin, 1993), as does a physical disconnection of these two areas in one hemisphere (Burcham et al., 1997).

Within the frontal cortex, the region of interest has been narrowed by more recent studies to the dorsolateral prefrontal cortex in humans and nonhuman primates (Dias et al., 1996a,b,

Received Sept. 24, 2002; revised 0ct. 23, 2002; accepted 0ct. 25, 2002.

This work was supported in part by National Institute on Aging Grant R03-AG17337. M.G.B. is an Alfred P. Sloan Research Fellow. We thank Drs. Elizabeth Gaffan and Joshua Rodefer for helpful comments on this manuscript.

Correspondence should be addressed to Dr. Mark G. Baxter, Department of Psychology, Harvard University, 906 William James Hall, 33 Kirkland Street, Cambridge, MA 02138. E-mail: mbaxter@wjh.harvard.edu.

Copyright $\odot 2003$ Society for Neuroscience $\quad 0270-6474 / 03 / 230676-06 \$ 15.00 / 0$
1997; Rogers et al., 2000) and the medial frontal cortex in rats (Birrell and Brown, 2000), which are believed to be functional homologs (Brown and Bowman, 2002). The medial frontal region studied by Birrell and Brown (2000) is a subset of the AGm region discussed above. These authors examined performance of rats in a nonspatial attentional set-shifting task based on the Wisconsin Card Sorting task (Roberts et al., 1994; Birrell and Brown, 2000). In this task, rats learned several two-choice discrimination problems between pairs of stimuli that differed in three sensory dimensions. The reward was consistently associated with one element of a single dimension. Once the initial discrimination was learned, a new problem was given with new exemplars of each dimension, with reward still associated with an element of the same relevant dimension. This problem is referred to as an intradimensional shift (IDS), because the new learning requires shifting attention to new stimuli, but the same perceptual dimension is relevant to solution to the problem. This is in contrast to an extradimensional shift (EDS), in which the relevant dimension changes from the previous problem. Rats (Shepp and Eimas, 1964; Birrell and Brown, 2000), monkeys (Dias et al., 1997), and humans (Owen et al., 1991, 1993; Gauntlett-Gilbert et al., 1999) all find solving new IDS problems easier than solving new EDS problems. Rats with lesions of the medial frontal cortex are impaired on EDS but not IDS problems (Birrell and Brown, 2000), a result that parallels findings in monkeys with lesions of the lateral frontal cortex (Dias et al., 1996a, 1997) and humans with resection of the frontal lobes (Owen et al., 1991).

Most studies of parietal cortex function in attention have used tests of spatial attention. Because of the interconnectedness of the prefrontal cortex and PPC and the recent demonstration of involvement of the PPC in aspects of nonspatial attention (Bucci et al., 1998), the aim of the present study was to study the effect of PPC damage on an attentional set-shifting task that engages the 
frontal cortex (Birrell and Brown, 2000). Rats were given neurotoxic lesions of the PPC, or a control surgery, and were postoperatively tested for the ability to establish and shift attentional sets. If the PPC and frontal cortex function as part of an attentional network, then we expected that PPC lesions, like medial frontal lesions, would impair attentional set-shifting.

\section{Materials and Methods}

Subjects. Twenty-four male Long-Evans rats (Taconic Farms, Germantown, NY), weighing 250-300 gm at the beginning of the study, were housed individually in $25 \times 45 \times 20 \mathrm{~cm}$ plastic cages. Testing was conducted during the light phase of a $12 \mathrm{hr}$ light/dark cycle (lights on at 8:00 A.M.). Before surgery and for 2 weeks afterward, rats were given access to food ad libitum. After this time they were maintained on a restricted feeding schedule with the amount of food administered contingent on their performance on the food-motivated task. A weight of $85 \%$ of ad libitum body weight served as a guideline. Water was always available ad libitum in the home cage. All experimental protocols were approved by the Harvard University Standing Committee on the Use of Animals in Research and Teaching.

Surgical procedures. Rats were randomly assigned to the lesion $(n=12)$ or control $(n=12)$ group. All subjects underwent precisely the same surgical procedure under aseptic conditions, except that control rats were given intracortical injections of sterile PBS, whereas lesion rats received injections of $0.09 \mathrm{M} \mathrm{NMDA}$ in PBS. Anesthesia was induced with Nembutal (sodium pentobarbital, 55 mg/kg, i.p.; NLS Animal Health, Owings Mills, MD). The anesthetized rat's head was shaved, and the rat was placed in a Kopf stereotaxic apparatus (David Kopf Instruments, Tujunga, CA) with the incisor bar set at $3.3 \mathrm{~mm}$ below the interaural plane. An incision was made through the scalp and fascia to expose the skull. Four holes were drilled on each side of the skull, at the following stereotaxic coordinates: anteroposterior, -4.0 and $-4.7 \mathrm{~mm}$; mediolateral, $+/-2.5$ and $+/-3.7 \mathrm{~mm}$; relative to bregma. One injection was made at each site, at a depth of $1.5 \mathrm{~mm}$ below the skull at the medial sites and $1.7 \mathrm{~mm}$ below the skull at the lateral sites. A total volume of $0.2 \mu \mathrm{l}$ was delivered at each injection site at a rate of $0.05 \mu \mathrm{l} / \mathrm{min}$. The needle was initially lowered to $2 \mathrm{~mm}$ below the skull to ensure that the dura mater was pierced and then raised to the injection site and left in place for $30 \mathrm{sec}$ before the injection, and for $2 \mathrm{~min}$ after. After the last injection, the wound was closed with absorbable sutures and antibiotic ointment containing a local anesthetic was applied. Rats received intraperitoneal injections of a dextrose/saline solution $(2 \mathrm{ml} / 100 \mathrm{gm})$ for rehydration, as well as an intramuscular injection of Banamine (flunixin megumine, 0.5 $\mathrm{mg}$ in $0.1 \mathrm{ml}$; NLS Animal Health) as an analgesic. An additional injection of Banamine was given $24 \mathrm{hr}$ after surgery. Rats were monitored during recovery from anesthesia and allowed a minimum of 2 weeks recovery time in the home cage before the onset of food restriction and behavioral testing.

Apparatus. Rats can be trained to dig in small bowls filled with sawdust to retrieve food reward (Wood et al., 1999). This experiment used terracotta flower pots as digging bowls, with an internal diameter and depth of $10 \mathrm{~cm}$. The food reward was a half of a Honey Nut Cheerio (General Mills, Minneapolis, MN). The rim of the pot could be scented with perfumed oils, and the scent was absorbed by the terracotta to produce a long-lasting odor. The odor was refreshed at the beginning of each testing session.

The test apparatus was a Plexiglas box measuring $50 \times 37.5 \times 25 \mathrm{~cm}$ with an opaque barrier separating one-third of the box from the rest (along the long axis of the box). On each trial, the two digging pots were placed adjacent to each other in the larger section of the box while the rat waited in the smaller section. The rat was given access to the pots by raising the divider, which was then put back down once the trial had begun.

Habituation. On the day before testing, rats were placed in the testing box, given access to two pots filled with the corn cob bedding used in their home cages, and baited with several Cheerios. The cups were rebaited continuously until the rats were digging reliably to retrieve the food rewards. This took $\sim 30 \mathrm{~min}$. At the end of this period, rats were trained on two simple discriminations (SDs) to a criterion of six consec-
Table 1. Example of a possible combination of stimulus pairs for a rat shifting from digging medium to odor as the relevant dimension

\begin{tabular}{|c|c|c|c|c|}
\hline \multirow[b]{2}{*}{ Discrimination } & \multicolumn{2}{|c|}{ Dimensions } & \multicolumn{2}{|c|}{ Exemplar combinations } \\
\hline & Relevant & Irrelevant & $S+$ & S- \\
\hline SD & Medium & & M1 & M2 \\
\hline \multirow[t]{2}{*}{$C D$} & Medium & Odor & M1/01 & $\mathrm{M} 2 / 02$ \\
\hline & & & M1/02 & $\mathrm{M} 2 / 01$ \\
\hline \multirow[t]{2}{*}{ IDS } & Medium & Odor & M3/03 & $\mathrm{M} 4 / 04$ \\
\hline & & & M3/04 & M4/03 \\
\hline \multirow[t]{2}{*}{ Reversal } & Medium & Odor & M4/03 & M3/04 \\
\hline & & & M4/04 & M3/03 \\
\hline \multirow[t]{2}{*}{ EDS } & Odor & Medium & 05/M5 & 06/M6 \\
\hline & & & $05 / \mathrm{M} 6$ & 06/M5 \\
\hline
\end{tabular}

Half of the rats switched from medium to odor, and half switched from odor to medium. The correct exemplar is shown in bold and can be paired with either exemplar from the irrelevant dimension. In the IDS and EDS, the stimuli were novel exemplars of each dimension.

utive correct trials: odor of rose versus sensual (Body Shop perfume oils, Wake Forest, NC) and medium of crumpled tissue paper versus shredded latex gloves. The order of the two SDs and positive stimuli were randomized across rats, with each lesioned rat matched with one normal control. These stimuli were not used again. The purpose of this preliminary phase was to acquaint the rats with the discrimination learning procedure, as well as to draw their attention to the two dimensions of the stimuli, which could be relevant for subsequent discrimination learning.

In all discrimination tasks, digging was defined as a vigorous displacement of the digging medium, because the reward was buried deeply within the pot. Thus, rats could investigate the digging medium with paws or snout before executing a "dig" response, and these choices were not scored. Thus, because the rats were allowed to sample the digging media by touch before digging, they could have used tactile or visual characteristics (or both) of the media to make their choices based on this dimension.

Testing paradigm. A trial began by raising the barrier to allow the rat access to the two digging pots, only one of which was baited. The first four trials of each discrimination constituted a discovery period in which the rat was allowed to dig in both pots, regardless of where he first began to dig. An error was recorded if the rat first dug in the unbaited pot. After the first four trials were completed, the rat was returned to the smaller section of the box if he dug first in the unbaited pot and was not permitted to find the food reward in the other pot. Testing continued until the rat reached a criterion of six consecutive correct trials.

In a single test session, rats performed a series of discriminations paralleling the procedure used by Birrell and Brown (2000) (Table 1). Initially, an SD between either two odors or two digging media was presented, followed by a compound discrimination (CD) with the same positive stimulus as the initial SD. In the $\mathrm{CD}$, a new dimension was introduced, but it was not a reliable predictor of the location of the food reward. An IDS was then presented; the IDS was another compound discrimination in which both the relevant and irrelevant stimuli were changed, but the relevant dimension (either odor or medium) remained the same. The IDS was then reversed, so that what was formerly the negative stimulus was changed to be the positive stimulus, with the irrelevant dimension still not predictive of the location of the reward. Finally, the rats were presented with an EDS in which the formerly irrelevant dimension became the relevant one, whereas the original dimension no longer held predictive value.

\section{Table 2. Stimulus pairs used}

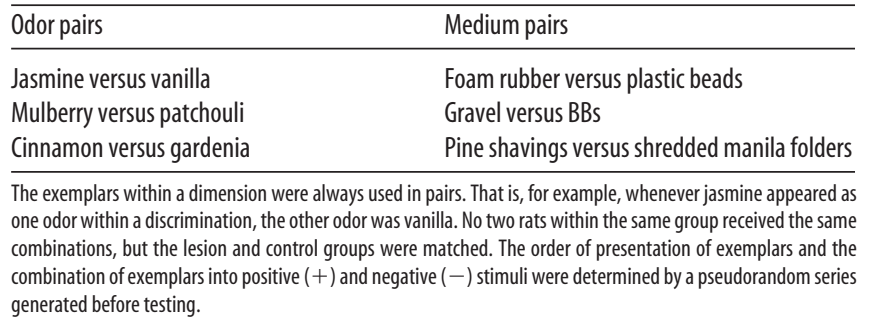
generated before testing. 
For instance, a pair of subjects could have experienced the following series of discriminations (Table 1): choose jasmine over vanilla, in a medium of neutral corn cob bedding (SD). Once the pair have chosen correctly for six consecutive trials, they must learn to choose jasmine over vanilla in cups filled with plastic beads or foam rubber, with the medium not linked to the location of the reward (CD). That is, a cup scented with jasmine could contain either beads or foam, with the other cup on each trial containing the other medium; the jasmine-scented cup would hold the food reward regardless of the medium it contained. Third, rats could be confronted with a new problem, the IDS, in which they must choose mulberry over patchouli, in cups filled with gravel or BBs, still ignoring the medium in which they dig. In this phase, although the stimuli are all novel, the same perceptual dimension (odor) is relevant for solving the discrimination. Next, the rats encounter the reversal phase, in which they learn to choose patchouli over mulberry, in the same media as the previous discrimination. Finally, they are presented with a third new problem, the EDS, in which they are presented with cups scented with, for example, either cinnamon or gardenia, and filled with pine shavings or shredded manila folders. However, in this instance, the reward would always be located in the cup filled with, for example, manila folders, regardless of whether it was scented with cinnamon or gardenia.

Half of the rats followed this order (shift from medium to odor as the relevant dimension), whereas half did the opposite (shift from odor to medium). The order of stimuli within a dimension was also randomized across subjects. There were too many possible pairings and orderings of stimuli to permit full counterbalancing, so the stimuli were assigned to pairs that were maintained across all subjects (e.g., when jasmine was the positive stimulus, vanilla was always the negative stimulus, and vice versa). The order of presentation of stimuli was counterbalanced to the greatest degree possible across rats. Particular care was taken to ensure that each exemplar was the positive stimulus of the EDS for one pair of subjects and the negative stimulus for another. With the exception of pair-matched controls, no two subjects were presented the stimuli in the same order.

Histology. After testing was complete, each rat was given a lethal dose of Nembutal (sodium pentobarbital, $100 \mathrm{mg} / \mathrm{kg}$, i.p.) and perfused transcardially with normal saline followed by $10 \%$ formalin in PBS at a flow rate of $18 \mathrm{ml} / \mathrm{min}$. The brains were then extracted and stored in a $20 \%$ sucrose-formalin solution. Brains were sectioned coronally on a freezing-sliding microtome at a thickness of $60 \mu \mathrm{m}$ (Fig. 1 contains sample photomicrographs of a lesion and a control brain). Every fourth section through the PPC was mounted on a gelatin-coated slide and stained with thionin for visualization of Nissl substance and then examined with a microscope to determine the magnitude and location of neurotoxic lesions (Fig. 2). One of the lesioned brains was destroyed during histological processing, and two lesioned rats had only unilateral damage; these three rats were excluded from our statistical analysis, bringing the number for the lesion group to nine.

\section{Results}

Rats with lesions to the PPC were selectively impaired on the EDS phase of the task (Fig. 3). An overall ANOVA with task phase as a within-subjects factor and both lesion and initial relevant dimension (medium or odor) as between-subjects factors revealed a main effect of task phase $\left(F_{(4,68)}=65.9 ; p<0.0005\right)$ as well as a task phase by lesion interaction $\left(F_{(4,68)}=12.7 ; p<0.0005\right)$. Post hoc analysis (unpaired $t$ tests on each phase) revealed a significant effect of lesion on the EDS phase only $\left(t_{(19)}=-4.28\right.$; $\left.p=0.0004\right)$, with no significant effect of lesion on any other task phase $(t<$ 1.01 ; $p$ values $>0.32$ ). Within-group analysis of only the control animals validated the task by revealing that for control rats, the
EDS is significantly more difficult to learn than the IDS $\left(t_{(11)}=\right.$ $-4.77 ; p=0.0006)$. Thus, although our task was slightly modified from the design described by Birrell and Brown (2000), the key feature (i.e., that the EDS discrimination is more difficult than the IDS) remains. There was no main effect of initial relevant dimension, nor were there any significant interactions of this effect with other factors ( $p$ values $>0.21$ ). Furthermore, the observation that PPC-lesioned rats are not impaired on the reversal, which is just as difficult as the EDS, also strongly suggests that they do not simply have an impairment in learning difficult discrimination problems.

It is interesting that the two rats with unilateral damage to the PPC performed outside the range of the rats with confirmed bilateral lesions on the EDS (lesion range, 13-19 trials to criterion; the two excluded cases completed the EDS in nine and 10 trials to criterion). This suggests that unilateral damage to the PPC is without effect on EDS performance.

\section{Discussion}

The interpretation of our results is straightforward: lesions of the PPC impaired the ability to shift attentional set from one perceptual dimension to another. The impairment cannot be explained in terms of an overall effect of the lesion on discrimination learning or the ability to attend to a perceptual dimension per se, because there were no differences between lesions and controls on the other four phases of our task. In particular, controls and lesions performed identically on the reversal phase of the task, which was of comparable difficulty to the EDS. Our results are consistent with those of a recent positron emission tomographic study of attentional set-shifting in humans, which found transient activation in the parietal cortex in subjects performing extradimensional relative to intradimensional shifts in addition to the prefrontal activation that was hypothesized (Rogers et al., 2000), as well as other functional neuroimaging studies that have found parietal activation induced by task switching (Gurd et al., 2002). We are not aware of any studies that have specifically examined performance of human patients with lesions of parietal cortex on attentional set-shifting or on related tasks such as the Wisconsin Card Sort, although we note that performance on the Wisconsin Card Sort test can be impaired by damage to sites outside the frontal lobe, including the parietal cortex (Anderson et al., 1991).

It is possible that the impairment we observed in the present study could be attributed to hippocampal damage that was unintentionally induced adjacent to the PPC. The hippocampus has been implicated in attentional function (Wall and Messier, 2001), albeit as an attentional monitor on the perceptual data that make up working memory rather than selection among dimensional 

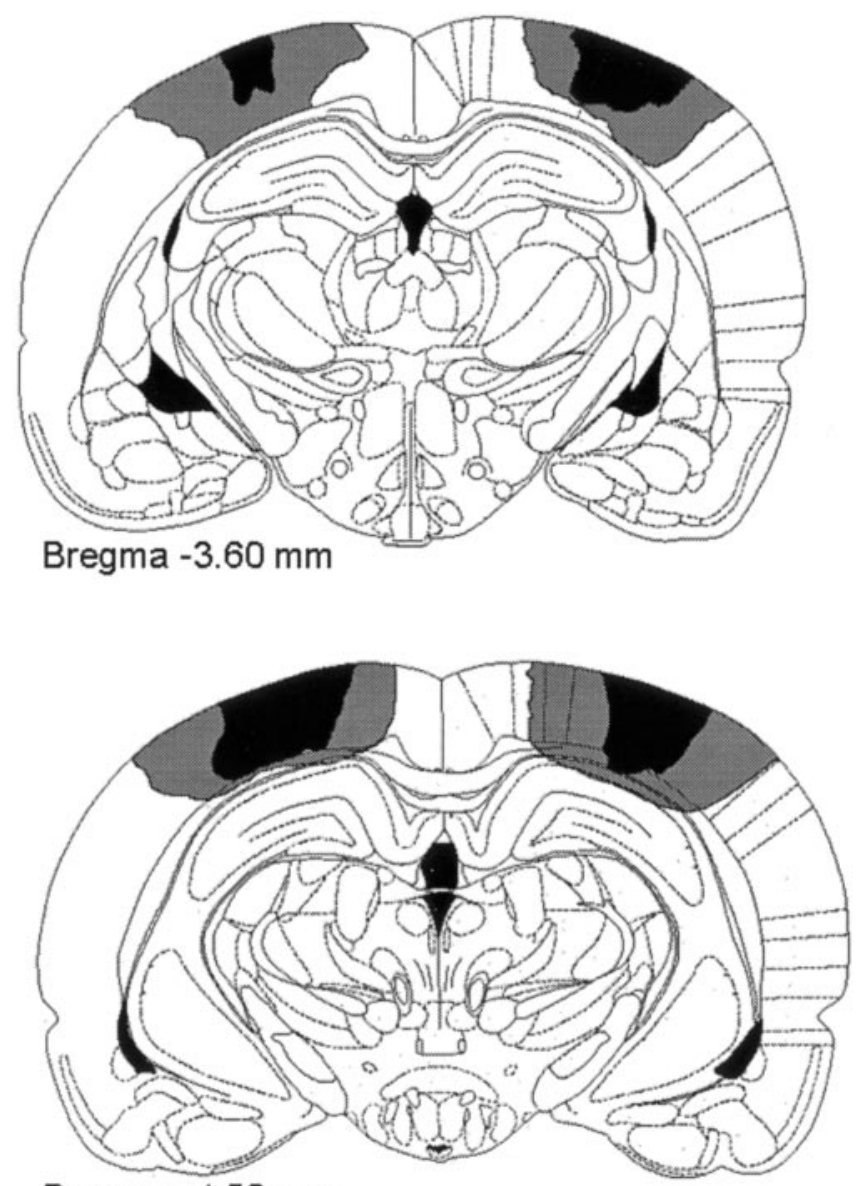

Bregma $-4.52 \mathrm{~mm}$

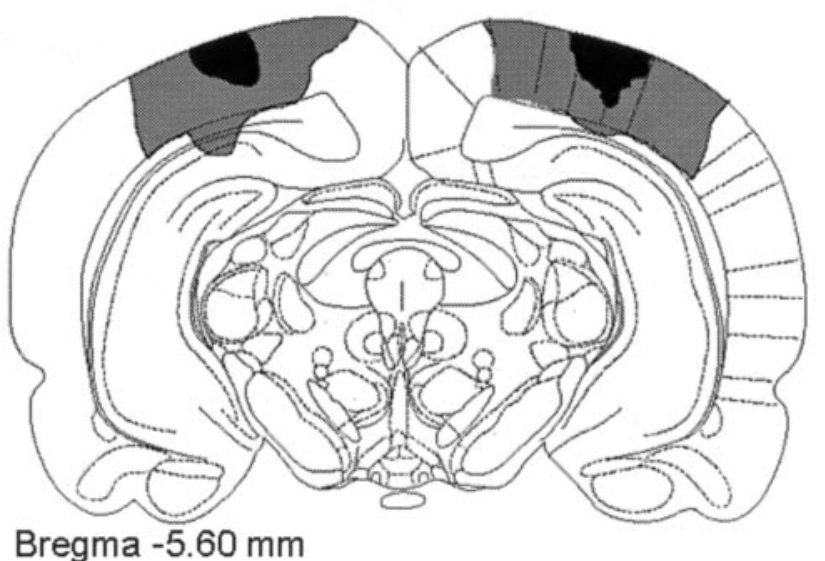

Figure 2. A series of coronal sections (adapted from Paxinos and Watson, 1998) at 3.6, 4.5, and $5.6 \mathrm{~mm}$ posterior to bregma. The area of damage common to most rats (at least 7 of the 9 rats included in the final data analysis) is shown in black, whereas the maximum area of damage to any rat is in gray.

sets. However, the hippocampal region implicated in this form of attention is ventral (Wall and Messier, 2001), and hippocampal damage in the present study was limited to the dorsal hippocampus. Furthermore, our recent study of the effects of aging on attentional set-shifting in rats found that spatial memory impairments as measured in the Morris water maze were not correlated with impairments on EDS (Barense et al., 2002), suggesting that the impairment in hippocampal function that occurs as part of

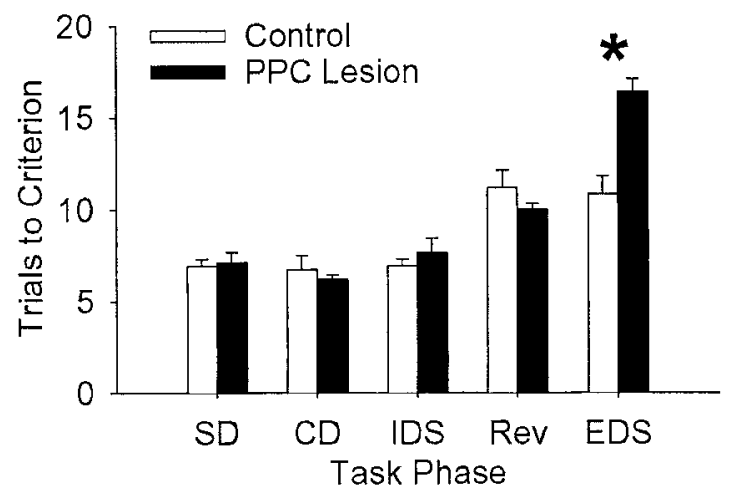

Figure 3. Rats with lesions to the PPC are selectively impaired on the EDS. The asterisk indicates a statistically significant difference between lesion and control rats, based on post hoc analysis $(p<0.05)$. Rev, Reversal.

normal aging, although sufficient to impair spatial learning, is not sufficient to produce impairment on the EDS. Finally, patients with unilateral temporal lobectomy or amygdalohippocampectomy are unimpaired on the EDS (Owen et al., 1991), and patient H.M., who had bilateral resection of the medial temporal lobes, including the rostral hippocampus, was unimpaired on the analogous Wisconsin Card Sort task (Milner et al., 1968).

Previous results indicate that other aspects of nonspatial attention are also impaired by disruption of PPC function, specifically by elimination of cholinergic projections to the PPC from the basal forebrain (Bucci et al., 1998). This study of Pavlovian associative learning can be contrasted with others that have focused instead on search strategies (Muir et al., 1996; Ward and Brown, 1997). Unilateral lesions of the PPC in rats produced no effect of response asymmetry on a visually cued response task, but there was a global increase in reaction time to stimuli presented contralesionally (Ward and Brown, 1997). Similarly, damage to parietal cortex in rats was without effect in a test of sustained attention (Muir et al., 1996); however, the lesions in this experiment were to the anterior parietal cortex rather than the posterior, so an effect of PPC damage on sustained attention (or vigilance) cannot be excluded. It is worth noting, however, that if any such effect existed, it was not sufficient to produce a generalized impairment in discrimination learning in the present study.

Any model of how a decision-based attentional task is performed must include at least two modules: one that gathers the relevant perceptual data and one that integrates that information into a behavioral strategy. Other data suggest that the PPC performs only the first of these two tasks. Bucci et al. (1998) studied attentional modulation of associative learning in a paradigm that did not involve the implementation of a behavioral strategy. Similarly, the result reported by Ward and Brown (1997), in which response latencies were increased but accuracy was unimpaired, suggests an effect at the level of perceptual modulation (increased latency to respond) rather than at the level of initiating a behavioral strategy. The selection of behavioral strategy is presumably the domain of the prefrontal cortex rather than the parietal cortex (Ragozzino et al., 1999a,b; Bussey et al., 2001, 2002; Gaffan et al., 2002). The present study, together with that of Birrell and Brown (2000), suggests that communication between the medial frontal cortex and PPC is essential to attentional function. This could be confirmed either by repeating the surgical disconnection method (i.e., both regions are left intact, but the axons running between them are severed) (Burcham et al., 1997) or by testing rats with crossed unilateral lesions of the two structures. 
Patients with parietal damage have difficulty in disengaging their attention from a cued spatial location to respond at a different location (Posner et al., 1984). Hence, the PPC may be necessary to disengage attention from stimuli that no longer accurately guide performance. The effect of PPC lesions on EDS learning in our study might thus be interpreted in this context as a difficulty in disengaging attention from the previously relevant perceptual dimension when attention to a new dimension is required to support accurate performance. Patients with damage to the frontal lobe also show this pattern of impairment (Owen et al., 1993).

Bucci et al. (1998) found that removal of cholinergic input to the PPC disrupts the ability to increase attention when a stimulus becomes an inconsistent predictor of an event that it previously consistently predicted, or when the magnitude of an expected reward decreases. These findings are consistent with theories of attentional modulation in Pavlovian conditioning that posit a loss of attention ("associability," or the capacity to learn about a stimulus) to stimuli that are consistent predictors of subsequent events or of reinforcement, with an increase in attention to stimuli that become inconsistent predictors of subsequent events (Pearce and Hall, 1980). Other theories of associative learning, which have been applied to discrimination learning and setshifting paradigms, suggest that attention is increased to stimuli that are good predictors of reinforcement and reduced to stimuli that are poor predictors of reinforcement (Mackintosh, 1975). Part of the conflict between these views is resolved by the notion that different forms of attention control performance and learning ("automatic" and "controlled" attentional processing, respectively) (Pearce and Hall, 1980). However, our results suggest that these views are not incompatible, at least at a neurobiological level. The phenomenon of a difference in learning EDS and IDS discriminations is predicted by Mackintosh's (1975) theory: attention is increased to stimuli in the relevant dimension and decreased to stimuli in the irrelevant dimension. When the EDS occurs, attention must be increased to the previously irrelevant dimension, thereby slowing acquisition of the EDS problem. Although this theory describes a type of attentional modulation that is entirely different from that posited by Pearce and Hall (1980), in both cases an increase in attentional processing is disrupted by PPC lesions. Another interpretation of our current result is that an impairment in increasing attention to the previously irrelevant dimension, rather than a failure to disengage attention from the previously relevant dimension, causes difficulty in learning EDS problems in PPC-lesioned rats. Thus, at least at a neural systems level, the PPC may be playing a similar role in modulating increases in attentional processing in different associative learning settings. Future experiments might try to experimentally dissociate these two possibilities.

The present work, combined with other experiments in rats (Burcham et al., 1997; Joel et al., 1997; Bucci et al., 1998), lends credence to the hypothesis that the PPC plays a general role in the deployment of attention to locations, objects, or perceptual streams that were previously unattended; in the disengagement of attention from locations, objects, or perceptual streams that were previously attended; or a combination of these. Although this concept is not novel as regards the direction of spatial attention, the present study suggests that the role of the PPC in these aspects of attention extends to a larger domain of perceptual processing than perhaps was previously appreciated. This may be useful in developing and testing theories of the neurobiological substrates of attentional processing within the parietal cortex.

\section{References}

Andersen RA, Asanuma C, Essick G, Siegel RM (1990) Corticocortical connections of anatomically and physiologically defined subdivisions within the inferior parietal lobule. J Comp Neurol 296:65-113.

Anderson SW, Damasio H, Jones DR, Tranel D (1991) Wisconsin Card Sorting Test performance as a measure of frontal lobe damage. J Clin Exp Neuropsychol 13:909-922.

Barense MD, Fox MT, Baxter MG (2002) Aged rats are impaired on an attentional set-shifting task sensitive to medial frontal cortex damage in young rats. Learn Mem 9:191-201.

Birrell JM, Brown VJ (2000) Medial frontal cortex mediates perceptual attentional set shifting in the rat. J Neurosci 20:4320-4324.

Brown VJ, Bowman EM (2002) Rodent models of prefrontal cortical function. Trends Neurosci 25:340-343.

Bucci DJ, Holland PC, Gallagher M (1998) Removal of cholinergic input to rat posterior parietal cortex disrupts incremental processing of conditioned stimuli. J Neurosci 18:8038-8046.

Burcham KJ, Corwin JV, Stoll ML, Reep RL (1997) Disconnection of medial agranular and posterior parietal cortex produces multimodal neglect in rats. Behav Brain Res 86:41-47.

Bussey TJ, Wise SP, Murray EA (2001) The role of ventral and orbital prefrontal cortex in conditional visuomotor learning and strategy use in rhesus monkeys (Macaca mulatta). Behav Neurosci 115:971-982.

Bussey TJ, Wise SP, Murray EA (2002) Interaction of ventral and orbital prefrontal cortex with inferotemporal cortex in conditional visuomotor learning. Behav Neurosci 116:703-715.

Corbetta M, Miezin FM, Shulman GL, Petersen SE (1993) A PET study of visuospatial attention. J Neurosci 13:1202-1226.

Corbetta M, Shulman GL, Miezin FM, Petersen SE (1995) Superior parietal cortex activation during spatial attention shifts and visual feature conjunction. Science 270:802-805.

Dias R, Robbins TW, Roberts AC (1996a) Dissociation in prefrontal cortex of affective and attentional shifts. Nature 380:69-72.

Dias R, Robbins TW, Roberts AC (1996b) Primate analogue of the Wisconsin Card Sorting Test: effects of excitotoxic lesions of the prefrontal cortex in the marmoset. Behav Neurosci 110:872-886.

Dias R, Robbins TW, Roberts AC (1997) Dissociable forms of inhibitory control within prefrontal cortex with an analog of the Wisconsin Card Sort Test: restriction to novel situations and independence from "online” processing. J Neurosci 17:9285-9297.

Gaffan D, Easton A, Parker A (2002) Interaction of inferior temporal cortex with frontal cortex and basal forebrain: double dissociation in strategy implementation and associative learning. J Neurosci 22:7288-7296.

Gauntlett-Gilbert J, Roberts RC, Brown VJ (1999) Mechanisms underlying attentional set-shifting in Parkinson's disease. Neuropsychologia 37:605-616.

Gurd JM, Amunts K, Weiss PH, Zafiris O, Zilles K, Marshall JC, Fink GR (2002) Posterior parietal cortex is implicated in continuous switching between verbal fluency tasks: an fMRI study with clinical implications. Brain 125:1024-1038.

Halligan PW, Marshall JC (1984) Spatial neglect. Hillsdale, NJ: Erlbaum.

Joel D, Weiner I, Feldon J (1997) Electrolytic lesions of the medial prefrontal cortex in rats disrupt performance on an analog of the Wisconsin Card Sorting Test, but do not disrupt latent inhibition: implications for animal models of schizophrenia. Behav Brain Res 85:187-201.

King VR, Corwin JV (1993) Comparisons of hemi-inattention produced by unilateral lesions of the posterior parietal cortex or medial agranular prefrontal cortex in rats: neglect, extinction, and the role of stimulus distance. Behav Brain Res 54:117-131.

Mackintosh NJ (1975) A theory of attention: variations in the associability of stimuli with reinforcement. Psychol Rev 82:276-298.

Mesulam M-M (1981) A cortical network for directed attention and unilateral neglect. Ann Neurol 10:309-325.

Milner B, Corkin S, Teuber H-L (1968) Further analysis of the hippocampal amnesic syndrome: 14-year follow-up study of H. M. Neuropsychologia 6:215-234.

Muir JL, Everitt BJ, Robbins TW (1996) The cerebral cortex of the rat and visual attentional function: dissociable effects of mediofrontal, cingulate, anterior dorsolateral, and parietal cortex lesions on a five-choice serial reaction time task. Cereb Cortex 6:470-481.

Owen AM, Roberts AC, Polkey CE, Sahakian BJ, Robbins TW (1991) Extradimensional versus intra-dimensional set shifting performance following 
frontal lobe excisions, temporal lobe excisions or amygdalohippocampectomy in man. Neuropsychologia 29:993-1006.

Owen AM, Roberts AC, Hodges JR, Summers BA, Polkey CE, Robbins TW (1993) Contrasting mechanisms of impaired attentional set-shifting in patients with frontal lobe damage or Parkinson's disease. Brain 116:1159-1175.

Paxinos G, Watson C (1998) The rat brain in stereotaxic coordinates, Ed 4. San Diego: Academic.

Pearce JM, Hall G (1980) A model for Pavlovian learning: variations in the effectiveness of conditioned but not of unconditioned stimuli. Psychol Rev 87:532-552.

Petersen SE, Robinson DL, Currie JN (1989) Influences of lesions of parietal cortex on visual spatial attention in humans. Exp Brain Res 76:267-280.

Posner MI, Walker JA, Friedrich FJ, Rafal RD (1984) Effects of parietal injury on covert orienting of attention. J Neurosci 4:1863-1874.

Ragozzino ME, Wilcox C, Raso M, Kesner RP (1999a) Involvement of rodent prefrontal cortex subregions in strategy switching. Behav Neurosci 113:32-41.

Ragozzino ME, Detrick S, Kesner RP (1999b) Involvement of the prelimbicinfralimbic areas of the rodent prefrontal cortex in behavioral flexibility for place and response learning. J Neurosci 19:4585-4594.

Reep RL, Chandler HC, King V, Corwin JV (1994) Rat posterior parietal cortex: topography of corticocortical and thalamic connections. Exp Brain Res 100:67-84.

Roberts AC, De Salvia MA, Wilkinson LS, Collins P, Muir JL, Everitt BJ, Robbins TW (1994) 6-Hydroxydopamine lesions of the prefrontal cor- tex in monkeys enhance performance on an analog of the Wisconsin card sort test: possible interactions with subcortical dopamine. J Neurosci 14:2531-2544.

Robinson DL, Bowman EM, Kertzman C (1995) Covert orienting of attention in macaques. II. Contributions of parietal cortex. J Neurophysiol 74:698-712.

Rogers RD, Andrews TC, Grasby PM, Brooks DJ, Robbins TW (2000) Contrasting cortical and subcortical activations produced by attentional-set shifting and reversal learning in humans. J Cogn Neurosci 12:142-162.

Selemon LD, Goldman-Rakic PS (1988) Common cortical and subcortical targets of the dorsolateral prefrontal and posterior parietal cortices in the rhesus monkey: evidence for a distributed neural network subserving spatially guided behavior. J Neurosci 8:4049-4068.

Shepp BE, Eimas PD (1964) Intradimensional and extradimensional shifts in the rat. J Comp Physiol Psychol 57:357-361.

Steinmetz MA, Constantinidis C (1995) Neurophysiological evidence for a role of posterior parietal cortex in redirecting visual attention. Cereb Cortex 5:448-456.

Wall PM, Messier C (2001) The hippocampal formation: orbitofrontal cortex circuit in the attentional control of active memory. Behav Brain Res 127:99-117.

Ward NM, Brown VJ (1997) Deficits in response initiation, but not attention, following excitotoxic lesions of posterior parietal cortex in the rat. Brain Res 775:81-90.

Wood ER, Dudchenko PA, Eichenbaum H (1999) The global record of memory in hippocampal neuronal activity. Nature 397:613-616. 